\title{
Cerebral localisation of a gastric malt lymphoma with partial response after chemotherapy
}

Sabbah Meriam ${ }^{1}$, Bibani Norsaf ${ }^{1}$, Trad Dorra ${ }^{1}$, Ouakaa Asma ${ }^{1}$, Jouini Raja $^{2}$, Ben Brahim Ehsen ${ }^{2}$, Elloumi Héla ${ }^{1}$ and Gargouri Dalila ${ }^{1}$

${ }^{1}$ Departement of gastroenterology. Habib Thameur Hospital, Tunis, Tunisia

${ }^{2}$ Departement of pathology. Habib Thameur Hospital, Tunis, Tunisia

\section{Clinical Image Representation}

A 37 years old woman presented upper gastrointestinal bleeding. Upper endoscopy and biopsy confirmed a gastric MALT lymphoma (Figure 1). She also presented headache without localization signs at neurological examination. Cerebral MRI showed an expanding tissue tumor process of the left choroid plexus associated with peri-lesional edema (Figure 2). The biopsy could not be realized because high risk of bleeding due to the localization of the tumor.

Lymphoma was classified stage IV E according to modified Ann Arbor Classification. She received chemotherapy (CHOP protocol). Endoscopic control showed a regression of the lymphoma with histopathological remission according to GELA score. Headache improved after treatment and control MRI (Figure 3) showed partial regression of the tumor after systemic treatment (after 3 months).

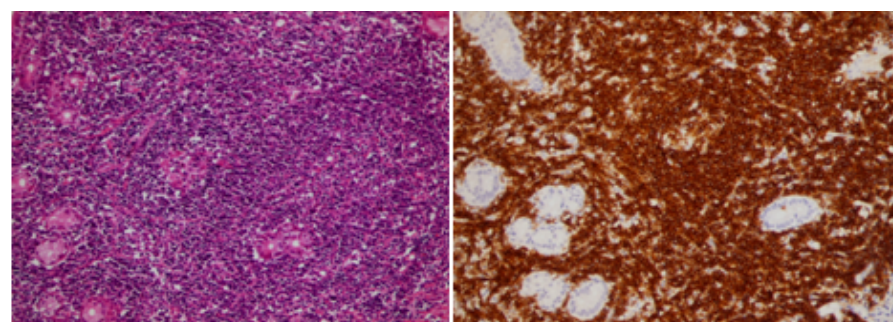

Figure 1. Initial histopathological examination: diffuse and abundant lymphoid infiltrate, realizing lymphoepithelial lesions: (Hematoxylin eosin x 200) and CD20 expression of tumor cells with immunohistochemistry ( $\mathrm{G}$ x 200).

Copyright: (C2018 Roever L. This is an open-access article distributed under the terms of the Creative Commons Attribution License, which permits unrestricted use, distribution, and reproduction in any medium, provided the original author and source are credited.

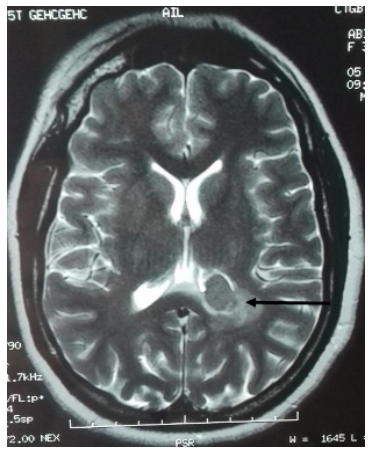

Figure 2. Initial MRI showing an expanding tissue tumor process of the left choroid plexus associated with a signal anomaly opposite: peri-lesional edema (arrow).



Figure 3. Control Computed tomography showing partial regression of the cerebral lesion (arrow).

*Correspondence to: Meriam Sabbah, Departement of Gastroenterology', Habib Thameur Hospital, Tunis, Tunisia, Tel: +216-71587452; E-mail: sabbah_ meriam@yahoo.fr

Received: July 06, 2018; Accepted: July 12, 2018; Published: July 16, 2018 